\title{
Establishing the presence of Cardiac Sub-Harmonics and its Importance in detecting Cardiac Abnormalities
}

\author{
Anandarup Mukherjee ${ }^{1}$, Disha Singhania ${ }^{2}$, Nidhi Pathak ${ }^{3}$, Parakh Khandelwal ${ }^{4}$ \\ 1,2,3,4 (Dept. of Electronics \& Communication Engg., University of Engineering \& Management, Jaipur, India)
}

\begin{abstract}
This paper presents a novel method for detecting cardiac abnormalities in a way which is both noninvasive and low cost. This method utilizes the presence of harmonics generated within the human heart as indicators of possible cardiac abnormalities such as cardiac blocks or cardiac valve dysfunctions. Our work is divided into two sections; firstly, the establishment of the fundamental frequency of the human heart along with its associated sub-harmonics and secondly, the presence of various harmonics in normal and abnormal cardiac signals which may serve as possible indicators to determine cardiac dysfunctions. Our method was applied on Phonocardiograph (PCG) signals of patients with various cardiac conditions.
\end{abstract}

Keywords: Curve Fitting, Fourier Series, Frequency Spectrum, Harmonics, Phonocardiogram.

\section{PhONOCARDiogram AND Its USe In CARdiaC Auscultation}

Heart sounds have been of great importance in the field of cardiography for a very long time. Studied and analyzed carefully, it helps in the detection of a variety of cardiac ailments. There have been many devices which have been used for the study of the heart sounds; most widely and commonly used amongst them is the

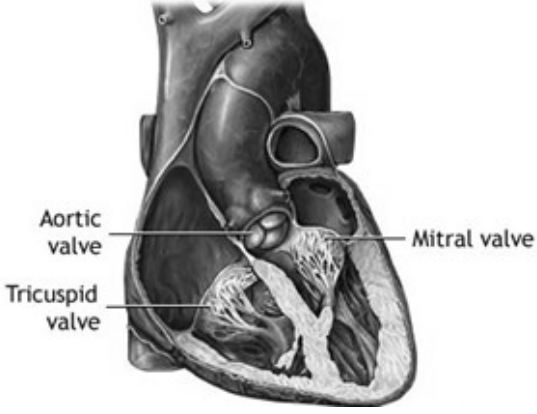

Figure 1: A cross section of the human heart depicting the positions of various valves.

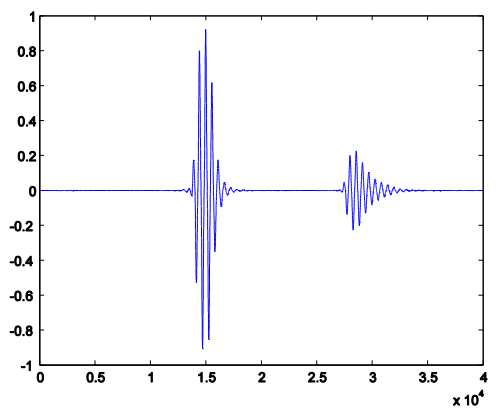

Figure 2: A PCG recording of normal S1-S2 complex.

stethoscope. At first, stethoscopes were purely mechanical and acoustic in nature which provided less amplification of sound. The advent of digital electronic stethoscopes made the detection of these sounds easier and efficient. New and more advanced versions of the electronic stethoscopes are regularly being launched into the market. These new versions of the stethoscopes come with monitors and provisions for displaying the heart sounds in analog form i.e., in the form of graphs. These graphs later on, can not only be viewed, but can also be processed and classified. This method of recording heart sounds is known as phonocardiography [1]. The graph generated is known as phonocardiogram. There are four types of heart sounds measured by phonocardiography namely systolic sound (S1), diastolic sound (S2), proto-diastolic sound (S3) and pre-systolic sound (S4).

The cardiac sounds occur in the heart due to opening and closing of the heart valves. The study of phonocardiogram of a heart reveals huge amount of information regarding the fitness of the heart and can lead to the detection of various cardiac diseases, if present. The heart sounds are classified in the consecutive subsections.

\subsection{First Heart Sound, S1}

This is a high frequency sound produced at the beginning of a systole i.e., contraction of the ventricles. It is caused due to the closure of atrio-ventricular valves. S1 is further divided into two sounds, M1 followed by T1. M1 is caused due to the closing of the mitral valve whereas T1 is produced due to the closing of the tricuspid valve. The blood flow from the atria to the ventricles raises the ventricular pressure causing the inlet valve to close. This closure prevents the reverse flow of blood into the atria and causes reverberation of blood which is detected as S1 in PCG. The S1 sound is depicted in figure 2. 


\subsection{SeCOnd Heart Sound, S2}

The second heart sound is yet another high frequency heart sound, produced at the end of a systole, which marks the beginning of diastole. It is caused due to the closure of aortic and pulmonary valves, also known as the semi-lunar valves. S2 comprises of two components A2 and P2. The blood flowing out from the left ventricle to the aorta causes a drop in the pressure of the left ventricle causing a reverse blood flow from the aorta to the ventricle. This flow is blocked by the closure of the aortic valve, causing the generation of A2. Similar mechanism is followed in the case of blood flow from the right ventricle to the pulmonary artery and the resulting blocking of the back flow from the artery to the right ventricle, causing P2. The S2 sound is depicted in figure 2.

\subsection{THIRD AND FOURTH HEART SOUNDS, S3 \& S4}

The third and fourth heart sounds S3 and S4 respectively, are both classified as low frequency sounds. S3 is heard after S2 and is of lower frequency than the S2 sound. The main cause of S3 is the movement of blood within the walls of the ventricles, causing reverberation and consecutively, its generation. It is generally

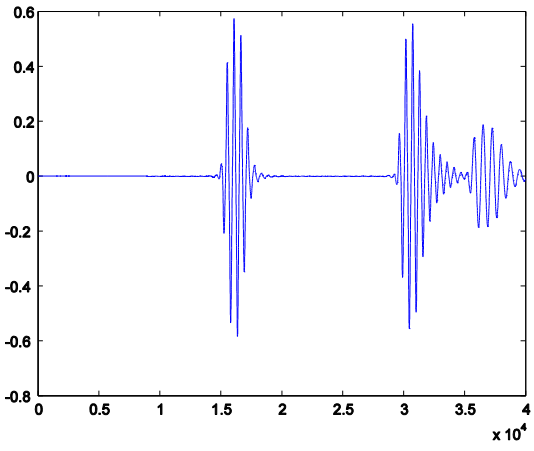

Figure 3: A PCG recording, depicting S1, S2 and S3 sounds (in sequential order).

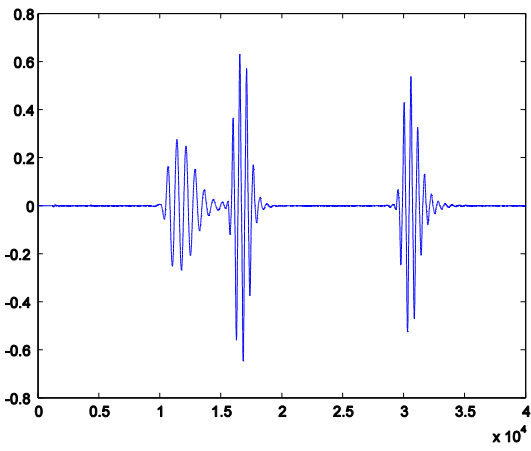

Figure 4: A PCG recording, depicting the heart sounds $S 4, S 1$ and S2 (in sequential order).

observed in athletes and is not harmful. However, it may indicate some serious heart disorders if found in aged patients. S4 is yet another low frequency sound which occurs just before S1. It is caused due to the forceful entry of blood into ventricles with stiffened walls. Rigid ventricular walls require the atria to pump blood into the ventricles with a force which is much greater than is normally required. This generates an extra sound S4 just before the beginning of systole. It may indicate a case of left ventricular disorder, restrictive cardiomyopathy and other such ailments. The heart sounds S3 and S4 are depicted in figure 3 and figure 4 respectively.

\section{Processing The Cardiac Sounds}

Various signal processing techniques were applied on the PCG signals, obtained from the University of Michigan, Heart Sound and Murmur Library [9], to extract meaningful information from them which, in turn could be universally applied to all PCG signals. Curve fitting on the signal was performed using Fourier series to obtain the fundamental frequency of the PCG complex at the instance of best fit. Consecutively, power spectrum analysis of the signal using Fourier transform was performed, to obtain the dominant frequency components of each PCG complex. Each of these methods is briefly explained in the following sub- sections.

\subsection{Curve FitTing}

Curve Fitting [2] [3] is the process of fitting a curve or line that fits best on the given series of data points. The main objective of this operation is to find the curve generating equation or the function along with its underlying parameters which fit best on the original set of data points. This function or equation can be used to find points anywhere along the curve. It can be done either by Interpolation or by Smoothing. Interpolation requires an exact fit on the data points, whereas Smoothing requires the creation of a smooth function or equation that nearly fits to the data. It is also known as regression analysis. Regression analysis focuses on the uncertainty of the curve using random errors.

The smooth curve fitting methods are Interpolation based on polynomials, Ratio of two polynomials, Fourier series expansion, Spline function, etc. For a single valued function, this method is based on the third order polynomial piecewise function whereas, for a multiple valued function, this method is based on third order pair of polynomials. 
Other than the above mentioned methods, another method is to produce a smooth curve. Here, a smooth curve is produced for a given set of unordered data just by knowing the starting and ending points of the data set. The polynomial curve fitting method is used for finding the boundary potential distribution function from the measured set of data. It is also used in modified Rice Algorithm. In many spectra, overlapping bands are present because of which many a times, the evaluation of band parameters, such as area, width and position, is not possible. The use of curve fitting helps in overcoming this problem as even with the presence of overlapping

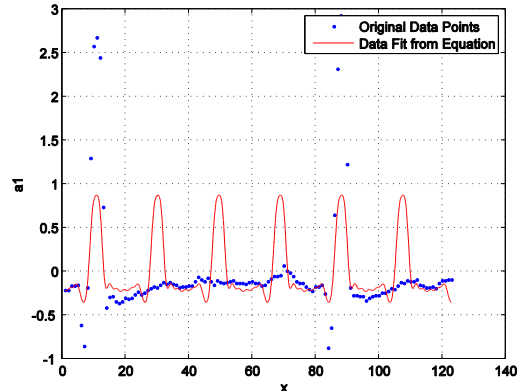

Figure 5: Plot of a curve fitting session with an overall bad fit $(R$-square $=0.1011)$

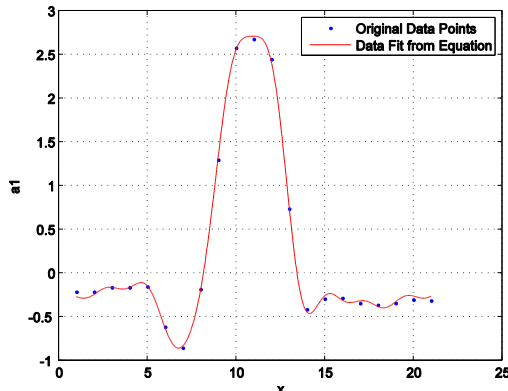

Figure 6: Plot of a curve fitting session with an overall excellent fit ( $R$-square $=0.9991)$

bands, the band parameters can be evaluated. This application of curve fitting is used in chemo-metrics and spectral analysis. The only condition for this method is that some information regarding the curve fitted spectrum is required. The parametric curve fitting is used in statistical data analysis pattern recognition, image processing and other applications. Sample curve fitting sessions using $8^{\text {th }}$ order Fourier Series expansion are given in figures 5 and 6 .

\subsection{FOURIER SERIES}

Fourier series provides an alternative way of representing a data or a waveform. A normal signal is represented as a function of time. It is very cumbersome to work on the generalized time domain form of the signal and extract much information from it. Fourier series transforms the signal information content from its time domain to its frequency domain. Instead of single amplitude as a function of time, a signal is expressed as the summation of its sine and cosine components. Working with frequency domain information is much easier and less complex than working with time domain information, which makes this method a powerful mathematical tool while dealing with periodic signals. This tool is widely used for the analysis of various signals and biological systems alike; some examples of the application domain of this tool include but are not limited to neurobiological rhythms, cardiac signals and others[4][5]. It isolates different frequencies present in the signal, providing an overview of how the system or signal is being affected by these frequency components and also the power spectrum of the signal [6]. The Fourier series can be mathematically generalized by equation 1 below.

$$
f(x)=\frac{a_{0}}{2}+\sum_{-\infty}^{\infty}\left(a_{m} \cos m x+b_{m} \sin m x\right)
$$

where, $a_{0}, a_{m}, b_{m}$ are Fourier series coefficients and $m$ is the fundamental frequency of the signal under Fourier series expansion. The property of the original wave can be recovered by using integration. This is possible due to the basic harmonics, which are sine and cosine functions given by Euler's formula in equation 2 .

$$
e^{2 \pi i \theta}=\cos (2 \pi \theta)+i \sin (2 \pi \theta)
$$

\subsection{Fourier Transform}

Fourier series finds its use in changing the time domain signals into frequency domain signals albeit; it only applies to periodic signals. There have been many questions about the application of Fourier series on biological systems due to the uneven nature of these systems. To transform a non-periodic signal from its time domain to its frequency domain, the concept of Fourier transform was introduced. Here, the period of the non-periodic signal is increased to approach infinity while maintaining its periodicity. This decomposed signal helps in understanding the distribution of various frequency components of the signal. The signal is represented in the form of its harmonics i.e., the sum of its sinusoidal components [7].

The following equation shows the mathematical expression for transferring a signal from its time domain to frequency domain: 


$$
F(\omega)=\int_{-\infty}^{\infty} f(t) e^{-i \omega t} d t
$$

Where, $F(\omega)$ is the signal in frequency domain, $f(t)$ is the time- domain signal to be transformed into frequency domain and $\omega$ is the fundamental frequency of the signal. To retrieve the original time- domain signal from its transformed frequency domain information, inverse Fourier transform is performed, which is given as:

$$
f(t)=\frac{1}{2 \pi} \int_{-\infty}^{\infty} F(\omega) e^{i \omega t} d \omega
$$

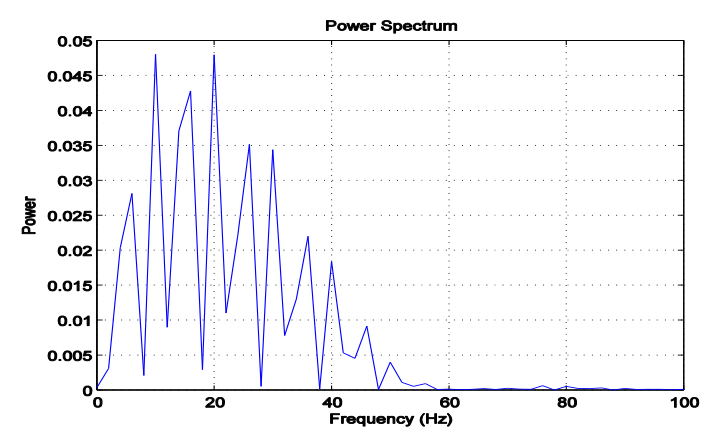

Figure 7: Figure depicting the plot of a Power Spectral Density (PSD) of a sample WSS signal

\subsection{Power Spectral Density}

Power Spectral Density (PSD) describes the distribution of the power of a signal with respect to its frequencies [8]. The power can be the actual power of the signal or in case of abstract signals; it is generally taken as the square of the signal. Squaring the signal is equivalent to the actual power obtained if the signal was applied, across a $1 \Omega$ load, as a voltage source. Since, a signal with non- zero average power is not square integrable, the Fourier Transform does not exist in this case. A simple alternative was provided by the WeinerKhinchin theorem [4], [5]. The PSD is considered as the Fourier Transform of the auto-correlation function $r_{x x}(\tau)$ of the signal, albeit, the signal is treated as a wide sense stationary (WSS) random process. Integrating the

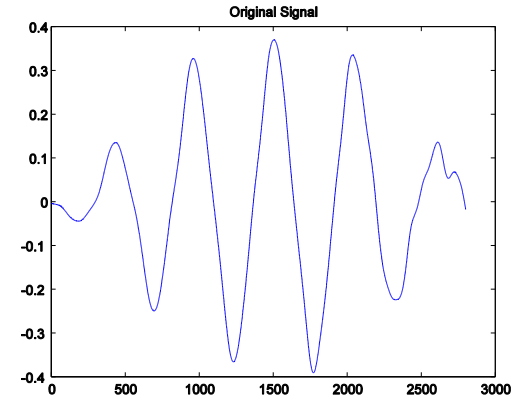

Figure 8: A normal S1 complex in a PCG signal
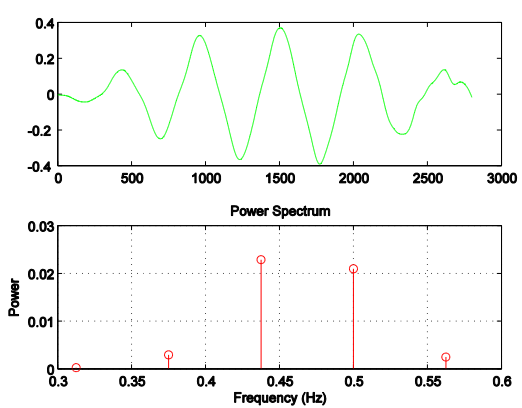

Figure 9: A normal S1 complex (Top) and its corresponding PSD (Bottom)

signal over a range of positive and negative frequencies, yields the power of that signal for that particular frequency band The PSD of a signal exists only for a wide sense stationary signal. In case of non- stationary signals, the auto-correlation function has to be a function of two variables, which rules out the existence of its PSD. Techniques similar to the Weiner- Khinchin theorem may be used to estimate a time varying spectral density as shown in figure 7.

For continuous- time signals, the Weiner- Kinchin theorem states that if $x$ is a WSS process such that the auto correlation function, $r_{x x}$ is defined in terms of the expectation value $E, r_{x x}(\tau)=E\left[x(t) . x^{*}(t)\right]$ exists and is finite at every time interval $\tau$, then there exists a monotone function $F(f)$ in the frequency domain $-\infty<f<\infty$, such that, $r_{x x}(\tau)=\int_{-\infty}^{\infty} e^{2 \pi i \tau f} d F(f)$ 
The PSD of a signal provides invaluable information about the frequency domain distribution of the signal strength, which can be used for designing Linear Time Invariant (LTI) filters to suppress or extract parts of a signal.

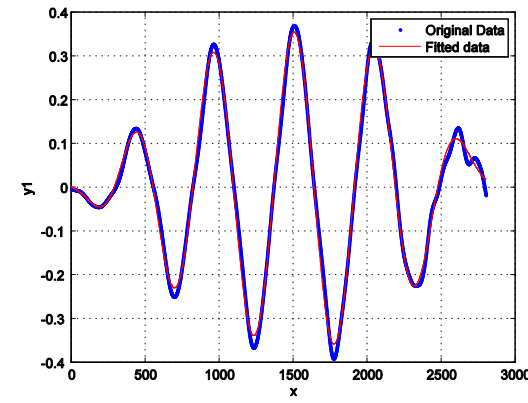

Figure 10: A plot showing the interpolation of the equation generated curve on the original data points.

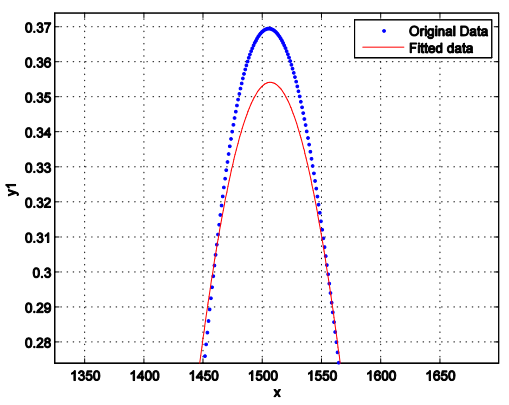

Figure 11: A magnified view of the interpolation of the generated curve on the original curve, plotted in Fig. 10

\section{METHODOLOGY \& OBSERVATIONS}

\subsection{DATABASE AND ITS DESCRIPTION}

The PCG signal database was obtained from the University of Michigan, Heart sound and Murmur library [9]. The database contains records of patients with various heart conditions. The PCG sounds are categorized primarily on the basis of the site of data acquisition, e.g. apex area, pulmonary area, aortic area, etc. The records show a variety of cardiac conditions such as murmurs and clicks, along with the presence of normal sounds. Two types of records were taken from the database viz., the normal S1 and S2 record and holo- systolic murmur, recorded at the apex region of the chest. This paper will henceforth, refer to the normal S1-S2 and the

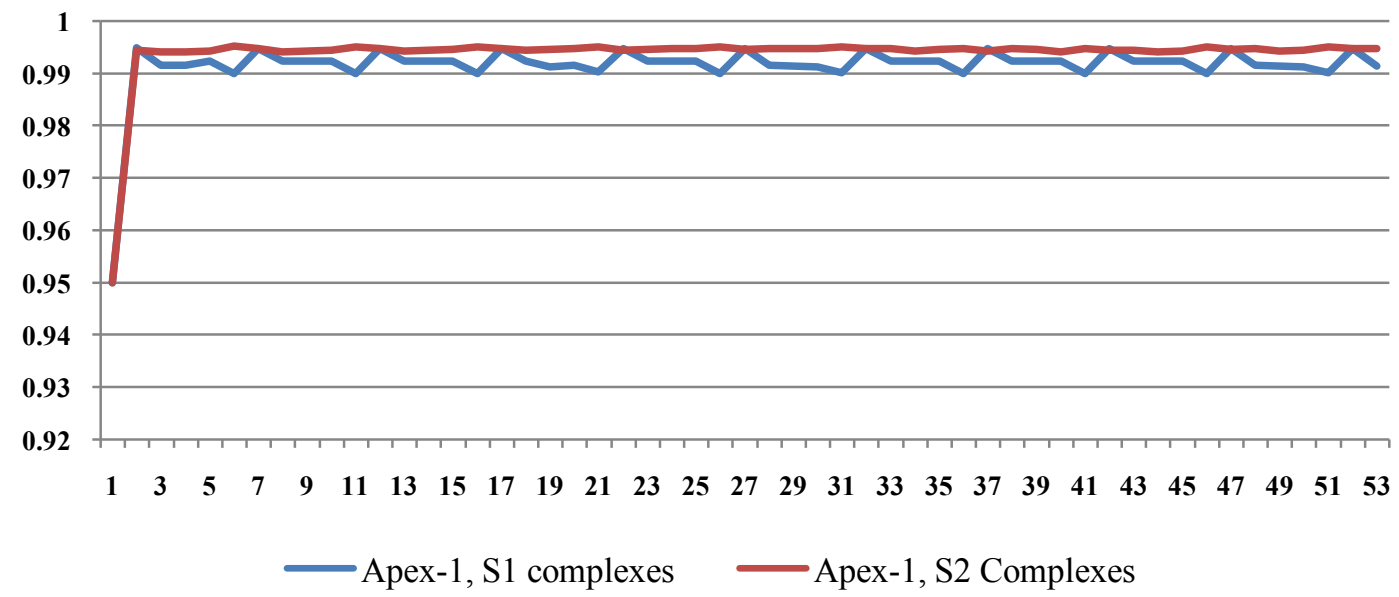

Figure 12: A plot of the $R^{2}$ values (Y-axis) for the corresponding complexes ( $X$-axis), obtained from Apex-1 record-set

Holo-systolic murmur record set as Apex-1 and Apex-9, respectively. All the files provided in the database are mp3 encoded with a bit rate of $128 \mathrm{kbps}$.

\subsection{Calculating the Fundamental frequency of a PCG COMPLex}

The records are first manually segregated into S1 and S2 complexes. Each of these complexes is decomposed into its constituent sinusoids using $8^{\text {th }}$ order Fourier series based curve fitting. The fit of the mathematically generated curve to the original signal gives us an approximate idea about the goodness of the curve generating equation in describing the constituents of the original signal. Figures 10 and 11 show the plots of the equation generated data after overlapping it on the original data points, to estimate the goodness of fit. The better the fit, the better is our mathematical formula for artificially generating the signal. The $R^{2}$ value is used to determine the goodness of the fit of the equation based curve to the original signal. Nearer the $R^{2}$ value is to unity, better is the fit of the curve. The $R^{2}$ values for all the complexes in both the records were calculated and it was found that the fit with the lowest $R^{2}$ value was 0.9593 , which actually indicates a very good fit. The plot 
of the $R^{2}$ values against its corresponding complex number for record Apex-1 (for both $\mathrm{S} 1$ and $\mathrm{S} 2$ complexes) is given in figure 12. It can be seen that the values are mostly above 0.95 . Having, established the goodness of fit of the $8^{\text {th }}$ order Fourier series to a PCG signal, the fundamental frequency of each complex is calculated. The Fourier expansion of a single PCG complex can be generalized by the following equation:

$$
\begin{aligned}
f(x)= & a_{0}+a_{1} \cos (x \omega)+a_{2} \cos (2 x \omega)+a_{3} \cos (3 x \omega)+a_{4} \cos (4 x \omega)+a_{5} \cos (5 x \omega)+a_{6} \cos (6 x \omega)+a_{7} \cos (7 x \omega)+ \\
& a_{8} \cos (8 x \omega)+b_{1} \sin (x \omega)+b_{2} \sin (2 x \omega)+b_{3} \sin (3 x \omega)+b_{4} \sin (4 x \omega)+b_{5} \sin (5 x \omega)+b_{6} \sin (6 x \omega)+b_{7} \sin (7 x \omega) \\
& +b_{8} \sin (8 x \omega)
\end{aligned}
$$

Here, $a_{0}, a_{1}-a_{8}, b_{1}-b_{8}$ are the Fourier coefficients and $\omega$ is the fundamental frequency of the signal. Since, the first objective of our study was established; the consecutive motive was to detect the harmonics in the original signal. The harmonics are the frequencies which are integer multiples of the fundamental frequency. For example, a sinusoidal signal with fundamental frequency $\omega$ will have $2 \omega, 3 \omega, 4 \omega, \cdots$ as its harmonics.

Table 1: The detected fundamental frequencies for $S 1$ and $S 2$ complexes for both record-sets Apex-1 and Apex-9

\begin{tabular}{|c|c|c|}
\hline Record & Complex & Fundamental frequency $\omega$ \\
\hline \multirow{2}{*}{ Apex-1 } & S1 & 0.001684 \\
\cline { 2 - 3 } & S2 & 0.001611 \\
\hline \multirow{2}{*}{ Apex-9 } & S1 & 0.001441 \\
\cline { 2 - 3 } & S2 & 0.002041 \\
\hline
\end{tabular}

Table-1 summarizes the calculated fundamental frequencies for all S1 and S2 complexes in both record sets Apex-1 and Apex-9. These fundamental frequencies correspond to the 52 individual complexes of S1 and S2 in each record set (Apex-1 and Apex-9).

\begin{tabular}{|c|c|c|c|c|c|}
\hline $\begin{array}{c}\text { Frequencies } \\
\text { Detected }\end{array}$ & S1 & S2 & $\begin{array}{c}\text { Sub- } \\
\text { Harmonics } \\
\text { (multiplying } \\
\text { factor) }\end{array}$ & $\begin{array}{l}\text { Sub- Harmonic_S1 } \\
\left(\omega_{S 1}=\mathbf{0 . 0 0 1 6 8 4} \mathbf{H z}\right)\end{array}$ & $\begin{array}{l}\text { Sub- Harmonic_S2 } \\
\left(\omega_{S 2}=\mathbf{0 . 0 0 1 6 1 1} \mathbf{H z}\right)\end{array}$ \\
\hline$f_{1}$ & 0.34375 & 0.34375 & $m_{1}=f_{1} / \omega$ & 204.1271 & 213.3768 \\
\hline$f_{2}$ & 0.375 & 0.375 & $m_{2}=f_{2} / \omega$ & 222.6841 & 232.7747 \\
\hline$f_{3}$ & 0.4065 & 0.4065 & $m_{3}=f_{3} / \omega$ & 241.3895 & 252.3277 \\
\hline$f_{4}$ & 0.4375 & 0.4375 & $m_{4}=f_{4} / \omega$ & 259.7981 & 271.5705 \\
\hline$f_{5}$ & 0.46875 & 0.46875 & $m_{5}=f_{5} / \omega$ & 278.3551 & 290.9683 \\
\hline$f_{6}$ & 0.5 & 0.5 & $m_{6}=f_{6} / \omega$ & 296.9121 & 310.3662 \\
\hline$f_{7}$ & 0.53125 & 0.53125 & $m_{7}=f_{7} / \omega$ & 315.4691 & 329.7641 \\
\hline$f_{8}$ & 0.5625 & 0.5625 & $m_{8}=f_{8} / \omega$ & 334.0261 & 349.162 \\
\hline
\end{tabular}

Tables $2 A \& B$ : list of the detected frequencies in all S1 and S2 complexes in Apex-1 and its equivalent subharmonic as calculated against the detected fundamental frequency.

\subsection{Establishing The existence OF Harmonics in a PCG SIGNAL}

This stage involves the PSD analysis of each complex in both records Apex-1 and Apex-9. The fundamental frequency calculated in the previous stage was used for checking the presence of harmonics in the PCG complexes by calculating the multiplying factor with respect to the detected fundamental frequency and the detected peaks in the PSD estimation stage. It was found that, for both records Apex-1 and Apex-9, there was a single fundamental frequency for both S1 and S2 complexes. This had variations only after four to five decimal places for the different record-sets and complexes, as is evident in Table-1.

Table 2A lists the frequency components detected in Apex-1 for both S1 and S2 complexes. Table 2B lists the multiplying factor, which was calculated with respect to the fundamental frequency of its corresponding complex. Tables 3A and 3B follows the above pattern, albeit, for record Apex-9. This multiplying factor is the sub-harmonic of the fundamental frequency. The multiplying factors in tables $2 \mathrm{~B}$ and $3 \mathrm{~B}$ are, thus, the subharmonics of the detected fundamental frequency. 
Table $3 A \&$ B: list of the detected frequencies in all S1 and S2 complexes in Apex-9 and its equivalent subharmonic as calculated against the detected fundamental frequency.

\begin{tabular}{|c|c|c|c|c|c|}
\hline $\begin{array}{c}\text { Frequencies } \\
\text { Detected }\end{array}$ & S1 & S2 \\
\hline$f_{1}$ & 0.375 & 0.375 \\
\hline$f_{2}$ & 0.4375 & 0.4375 \\
\hline$f_{3}$ & 0.5 & 0.5 \\
\hline$f_{4}$ & 0.5625 & 0.5625 \\
\hline
\end{tabular} \begin{tabular}{|c|c|c|}
$\begin{array}{c}\text { Sub- } \\
\text { Harmonics } \\
\text { (multiplyin }\end{array}$ & $\begin{array}{c}\text { Sub-Harmonic_S1 } \\
\left(\omega_{S 1}=\mathbf{0 . 0 0 1 4 4 1} \mathbf{H z}\right)\end{array}$ & $\begin{array}{c}\text { Sub-Harmonic_S2 } \\
\left(\omega_{S 2}=\mathbf{0 . 0 0 2 0 4 1 H z}\right)\end{array}$ \\
\hline$n_{1}=f_{1} / \omega$ & 260.2359 & 183.7335 \\
\hline$n_{3}=f_{3} / \omega$ & 303.6086 & 214.3557 \\
\hline$n_{4}=f_{4} / \omega$ & 346.9813 & 244.978 \\
\hline
\end{tabular}

\section{CONCLUSION}

It was observed from Table 1 that the fundamental frequencies for the complexes S1 and S2 were almost same up-to four decimal places in the record-set apex-1, whereas the values of fundamental frequencies for S1 and S2 complexes in record-set apex-9 were same up-to two decimal places.

Tables-2a and $2 \mathrm{~b}$ show the sub-harmonics detected for apex-1 S1 and S2 complexes. A total of 8 frequencies were detected in the power spectrum for each S1 and S2 complexes. The difference between successive S1 sub-harmonics is approximately 18 whereas for successive S2 sub- harmonics are approximately 19. Similarly, tables- $3 a$ and $3 b$ show the sub- harmonics detected for apex-9 or the record with holo-systolic murmur. A total of 4 frequencies were registered on the power spectrum of this record-set. The difference between successive S1 sub- harmonics was 43.37 and S2 sub- harmonics was 30.622.

This wider difference between successive sub- harmonics in apex-9 (holo-systolic murmur) can be attributed to the abnormal nature of the signal, due to the presence of murmurs. This difference was observed in the S1 and S2 complexes, without taking into account, the actual murmur signals lying between the S1 and S2 complexes. This system can be used for detecting or predicting heart valve abnormalities cheaply, as this system relies only on the powerful S1 or S2 sounds (which are easiest to detect). The non dependence of this system on the need for precision equipments for detecting the faint murmur sounds makes this method very cheap. Our future work will comprise mainly on the implementation of this method on actual hardware and testing it on patients with valve dysfunctions.

Books:

\section{REFERENCES}

[1] Howard B. Sprague, M.D., Patrick A. Ongley, M.D., “The Clinical Value of Phonocardiography”, Circulation. 1954;9:127-134

[2] Sandra LachArlinghaus, PHB Practical Handbook of Curve Fitting. CRC Press, 1994.

[3] William M. Kolb. Curve Fitting for Programmable Calculators. Syntec, Incorporated, 1984

[4] C. Chatfield (1989). The Analysis of Time Series-An Introduction (fourth ed.). Chapman and Hall, London. pp. 94-95. ISBN 0-41231820-2.

[5] Hannan, E.J., "Stationary Time Series", in: John Eatwell, Murray Milgate, and Peter Newman, editors, The New Palgrave: A Dictionary of Economics. Time Series and Statistics, Macmillan, London, 1990, p. 271.

Journals:

[6] E. O. Attinger, A. Anné, D. A. MacDonald, "Use of fourier series for the analysis of biological systems", Biophysical journal, Vol. 6, 1996.

[7] Jennifer Urbano Blackford, Ronald M. Salomon, Neils G. Waller, "Detecting change in biological rhythms: A multivariate permutation test approach to fourier transformed data", Chronobiology international, 2009, Vol. 26, No. 2, Pg 258-81.

[8] David R. Brillinger, "the annals of statistics", 2002, Vol. 30, No. 6, pg 1595-1618.

\section{Online Resources:}

[9] University of Michigan, Heart Sound and Murmur Library, http://www.med.umich.edu/lrc/psb/heartsounds/. 


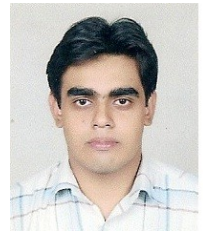

\section{About THe Authors}

Anandarup Mukherjee (M' 12). The author is a member of IEEE since 2012. He is currently attached as an Asst. Professor in the department of Electronics \& Communications Engg. at the University of Engineering \& Management, Jaipur. The author holds a Masters' degree in Electronics \& Communications Engg, with specialization in Microelectronics \& VLSI design. His research interests include Biomedical signal processing, especially cardiac signal processing, embedded systems and VLSI.

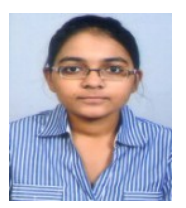

Disha Singhania is a third year student pursuing her Bachelor's degree in the field of Electronics and Communication Engineering from the University of Engineering and Management, Jaipur Rajasthan, India. Her area of interest includes Embedded Systems, VLSI, statistical Bio-Medical Signal Processing, Image Processing and Robotics. She regularly works on hardware as well as software based application of signal processing as well as image processing and aims to become a researcher.

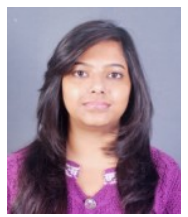

Nidhi Pathak is a fourth year student pursuing her Bachelor's degree from University of Engineering \& Management, Jaipur, India, in the department of Electronics \& Communication Engineering. Her area of interest includes Bio-Medical Signal Processing, statistical analysis and VLSI. She regularly contributes research papers and articles to various conferences and journals.

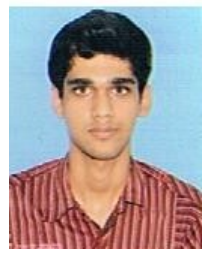

Parakh Khandelwal is a third year student pursuing his Bachelor's degree in the field of Electronics and Communication Engineering from the University of Engineering and Management, Jaipur Rajasthan, India. His area of interest includes Embedded Systems, VLSI, Bio-Medical Signal Processing and Robotics. He regularly works on hardware as well as software based application of signal processing and aims to be a researcher. 